\title{
Sub-pixel resolving optofluidic microscope for on-chip cell imaging $\dagger$
}

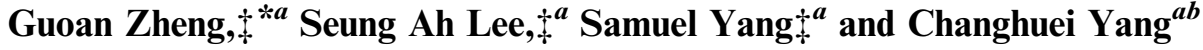

\author{
Received 20th July 2010, Accepted 24th August 2010 \\ DOI: 10.1039/c0lc00213e
}

\begin{abstract}
We report the implementation of a fully on-chip, lensless, sub-pixel resolving optofluidic microscope (SROFM). The device utilizes microfluidic flow to deliver specimens directly across a complementary metal oxide semiconductor (CMOS) sensor to generate a sequence of low-resolution (LR) projection images, where resolution is limited by the sensor's pixel size. This image sequence is then processed with a pixel super-resolution algorithm to reconstruct a single high resolution (HR) image, where features beyond the Nyquist rate of the LR images are resolved. We demonstrate the device's capabilities by imaging microspheres, protist Euglena gracilis, and Entamoeba invadens cysts with sub-cellular resolution and establish that our prototype has a resolution limit of 0.75 microns. Furthermore, we also apply the same pixel super-resolution algorithm to reconstruct HR videos in which the dynamic interaction between the fluid and the sample, including the in-plane and out-of-plane rotation of the sample within the flow, can be monitored in high resolution. We believe that the powerful combination of both the pixel super-resolution and optofluidic microscopy techniques within our SROFM is a significant step forwards toward a simple, cost-effective, high throughput and highly compact imaging solution for biomedical and bioscience needs.
\end{abstract}

\section{Introduction}

There is a growing interest in miniaturized microscopy systems. ${ }^{1-12}$ Compact, inexpensive and portable imaging systems can fulfill many needs in biological research, point-of-care analysis and field diagnostics. For example, an on-chip microscope, produced at scale at existing semiconductor foundries and capable of imaging blood cell or parasite morphology in high resolution, would bring affordable healthcare diagnostics to less developed populations in rural settings, where it is too costly to deploy expensive conventional microscopes and skilled technicians.

In recent years, two classes of on-chip microscopy methods have been extensively reported to address these needs. The first method, optofluidic microscopy (OFM), ${ }^{1}$ scans target objects across an aperture array using a microfluidic flow. The second method, inline holography, ${ }^{2,3}$ computationally renders images of target objects from interferometry measurements of the objects' scattered light field. Both methods achieve resolutions better than the sensor pixel size, but each has its respective strengths and tradeoffs. The inline holography approach works well with samples prepared on glass slides and represents a good direct alternative to conventional microscopy. The OFM approach works directly with fluid samples and has the potential for integration with streamlined and high throughput microfluidic systems. The OFM achieves its highest sharpness at the floor

\footnotetext{
${ }^{a}$ Department of Electrical Engineering, California Institute of Technology, MC 136-93, 1200 E. California Blvd, Pasadena, CA, 91125, USA. E-mail: gazheng@caltech.edu; Fax: +1(626)3953786; Tel: +1(626)3953944

${ }^{b}$ Department of Bioengineering, California Institute of Technology, MC 136-93, 1200 E. California Blvd, Pasadena, CA, 91125, USA

$\uparrow$ Electronic supplementary information (ESI) available: Detailed calculation of the motion vector and an algorithm for motion estimation, reconstructed high resolution video of Entamoeba invadens cysts. See DOI: 10.1039/c0lc00213e

\$ These authors contributed equally to this work.
}

of the microfluidic channel and its resolution is limited by the aperture size. ${ }^{4,13}$ The resolution degrades as a function of sample-to-floor separation. ${ }^{4}$ Inline holography's resolution is directly related to the SNR of the capture image and sensor pixel size.

Recently, Bishara et al. combined inline holography with a multiframe pixel super-resolution approach to effectively shrink their image pixel size and thereby attain higher resolution images. ${ }^{14}$ Multiframe pixel super-resolution approaches rely on combining information from multiple sub-pixel-shifted low resolution (LR) images to create a single high resolution (HR) image. Effectively, pixel super-resolution approaches take advantage of over-sampling in the time domain (capturing a sequences of images rather than a single image) to compensate for under-sampling in the spatial domain, for which the resolution is limited by the image sensor pixel size. To capture such a sequence of shifted LR images, either a fixed image sensor could image a spatially translating object, or a translating image sensor could image a fixed object. In either case, the success of the applied pixel super-resolution algorithm for HR image restoration depends critically on the precise control over and knowledge of the relative sub-pixel shifts between subsequent LR images. In the inline holography experiment reported in ref. 14, a mechanical stage was employed $10 \mathrm{~cm}$ above the sample to generate these sub-pixel shifts by precisely translating an optical fiber coupled with a light source.

Interestingly, not only is this multiframe pixel super-resolution approach adaptable for use in OFM as well, but it also greatly simplifies the microscopy scheme. More specifically, the microfluidic flow of the samples across the sensor suffices for generating sub-pixel shifts and precludes the need for an additional precision scanning setup. Furthermore, the resolution enhancement provided by the aperture arrays employed in current OFM system can be replaced by the enhancement provided by applying the pixel super-resolution algorithm. Without the aperture 
arrays, the OFM would be an even simpler on-chip microscopy design, consists solely of a microfluidic channel emplaced upon a commercial image sensor chip.

In this paper, we report on the implementation of a sub-pixel resolving optofluidic microscope (SROFM) based on this concept of applying pixel super-resolution algorithms in a new and simplified OFM scheme. We first give an overview of the entire imaging scheme, and then define in detail some of the more critical hardware design requirements and image processing techniques used, including methods for sub-pixel-shift estimation and a shift-and-add pixel super-resolution algorithm. We then present SROFM images of microspheres, protist Euglena gracilis, and Entamoeba invadens cysts and characterize the resolution limit of SROFM. Next, we discuss some of the advantages of SROFM, including the ability to image various different threedimensional projections of rotating samples, and demonstrate our results in reconstructing high resolution video, where the time-varying interaction between the sample and the microfluidic flow is shown. Finally, we conclude with a discussion on possible biomedical and bioscience applications for SROFM.

\section{Results and discussion}

The SROFM technique involves flowing a sample within a microfluidic channel directly above the surface of a CMOS image sensor (Fig. 1(a)). A sequence of pixel-size-limited low resolution (LR) direct-projection images of the sample, in which the sample within subsequent LR images is shifted by a sub-pixel amount, is captured and processed using a pixel super-resolution algorithm to produce a single high resolution (HR) image. The microscopy scheme is based on the integration of a microfluidic channel onto a CMOS image sensor. Compared to the aperturebased OFM technique described earlier, SROFM does not require a metal layer or an aperture array.
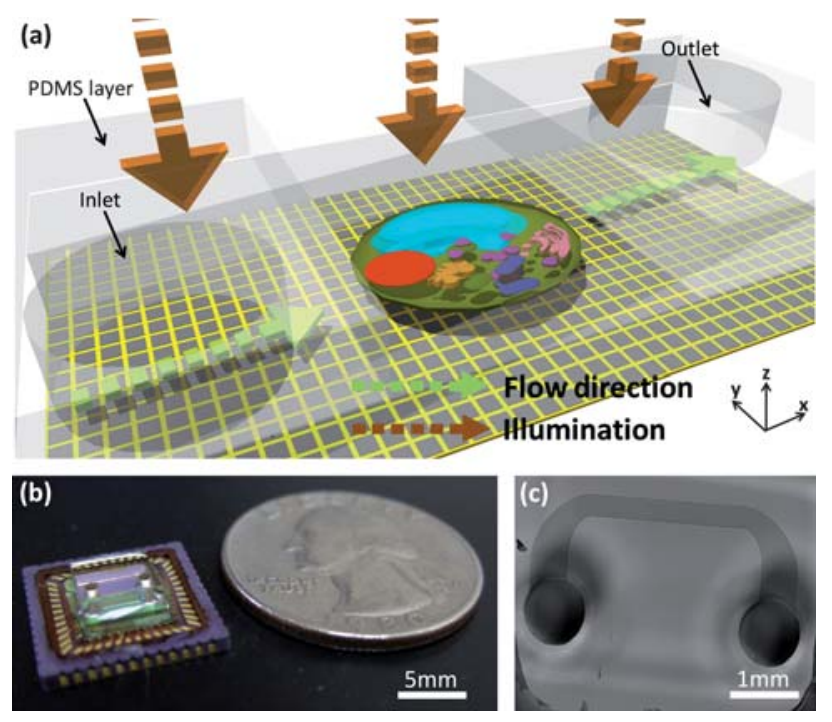

Fig. 1 (a) Schematic of the proposed device. A sample, depicted as a cell in the figure, is flown in a microfluidic channel on top of an image sensor and the LR projection images are recorded. (b) Photograph of an actual sub-pixel resolving optofluidic microscope (SROFM) device. (c) Image of the microfluidic channel directly captured by the SROFM image sensor.
The SROFM hardware is compact, measuring approximately $1.5 \mathrm{~cm} \times 1.5 \mathrm{~cm}$ in size (Fig. 1(b)), and consists of a polydimethyl-siloxane (PDMS) microfluidic channel (Fig. 1(c)) of width between $50 \mu \mathrm{m}$ and $300 \mu \mathrm{m}$ and height between $15 \mu \mathrm{m}$ and $27 \mu \mathrm{m}$, with an inlet and outlet hole, bonded directly to an inexpensive, commercially available CMOS image sensor (Aptina MT9T001), with $3.2 \mu \mathrm{m}$ pixel size. We designed the channel height to be just slightly larger than the dimensions of the samples being imaged and removed the color filter and microlens layer on the sensor surface to ensure the samples flowed as close as possible to the sensor's surface. The microfluidic channel was attached to the CMOS sensor at an angle between 10 to 30 degrees with respect to the $\mathrm{x}$ direction of the CMOS pixel grid to ensure that the captured LR image sequence contained the necessary sub-pixel translations in both the $\mathrm{x}$ and $\mathrm{y}$ directions. Our choice of angle struck a balance between using a smaller angle with an imaging-area-of-interest with fewer pixel rows (and hence higher frame rate) and using a larger angle to obtain sufficient displacement along the y-direction with fewer frames. Our experiments were conducted under light intensity of $1.2 \mathrm{~W} / \mathrm{m}^{2}$ (comparable to room light) provided by a conventional LED lamp and we employed a single frame exposure time of $0.3 \mathrm{~ms}$.

For imaging, a liquid containing an adequate concentration of target objects is introduced into the channel from the inlet and the pressure difference between the inlet and outlet and capillary action induce a flow, delivering the samples across the sensor pixel grid of the CMOS sensor. Samples were typically imaged at a flow velocity of $200 \mu \mathrm{m} / \mathrm{s}$, with a frame rate of $500 \mathrm{fps}$ and a field-of-view (FOV) of $250 \mu \mathrm{m}$ by $3 \mathrm{~mm}$. Note that the ultimate throughput limitation of SROFM is the speed of the CMOS image sensor. Even with our sensors (Aptina MT9T001), assuming the same fixed data transfer rate, with a more restricted FOV of $50 \mu \mathrm{m}$ by $100 \mu \mathrm{m}$ and maximum frame rate of $75000 \mathrm{fps}$, our current device could image a continuous stream of cells at a rate of $1300 \mathrm{HR}$ images/s with each image possibly containing multiple cells; to further increase the throughput of our device, we could use a high speed global shutter CMOS image sensors.

In multiframe pixel super-resolution techniques, the quality of the reconstructed HR image depends largely on knowledge of the precise sub-pixel shifts between subsequent images, collectively termed the motion vector of the LR image sequence. While the motion vector of a LR image sequence is in theory a known set of values in pixel super-resolution schemes involving precision scanning stages or actuators, implementation of a compact, microscanning system would not only be difficult and costly, but also prone to structural stability issues that could affect the accuracy of the motion vector. In contrast, the motion vector in SROFM can be estimated, quite accurately, by analyzing the rough position of the sample across the LR image sequence since the flow of the sample is highly stable within the microfluidic channel (low Reynolds number). For the simplest assumption of a sample flowing at constant velocity, the motion vector can be calculated from the location of the sample in the first and last frames. However, in many cases, the sample's motion is not strictly uniform or purely translational due to the geometry of the sample itself, defects in the microfluidic channel or changes in the flow rate. To compensate for this non-uniform motion, we developed several fast image processing techniques, discussed in 
the supplementary material S1, to estimate the motion vector from the LR sequence.

Once the motion vector of the LR image sequence is calculated, a shift-and-add pixel super-resolution algorithm can be applied to reconstruct a single HR image. ${ }^{15}$ Such an algorithm consists of shifting each LR image by the relative sub-pixel shift given by the computed motion vector and adding them all together to fill a blank HR image grid of enhancement factor of $n$, where each n-by-n pixel area of the HR image grid corresponds to a single 1-by-1 pixel area of the LR image grid. The wiener deconvolution method is then used to remove pixel blurring in the final HR image. Fig. 2 shows both LR and HR SROFM images of several microscopic objects: a $15 \mu \mathrm{m}$ microsphere, protist Euglena gracilis $(\mathrm{d} \sim 15 \mu \mathrm{m})$ and Entamoeba invadens cysts $(\mathrm{d} \sim 25 \mu \mathrm{m})$. Taking into account the Nyquist sampling criteria and image sensor pixel size of $3.2 \mu \mathrm{m}$, the resolution limit of each LR image should be $6.4 \mu \mathrm{m}$. Note that each individual LR image contains very little spatial information about the sample other than its rough location and size, but each HR image is clearly improved in resolution. Conventional microscope images taken with a 20x objective are also shown for comparison.

To establish the extent of resolution improvement, we imaged a solution of $0.5 \mu \mathrm{m}$ microspheres. Fig. 3 shows the reconstructed HR image of a $0.5 \mu \mathrm{m}$ sphere with the enhancement factor of 10 and 13. The centers of these microspheres can clearly be resolved and identified with the full width at half maximum (FWHM) of $0.80 \mu \mathrm{m}$ and $0.65 \mu \mathrm{m}$. The second data set implies that our prototype has a resolution limit of $\sim 0.75 \mu \mathrm{m}$ (adjacent particles can be resolved as long as they are 3 or more HR pixels apart). Note that the resolution of a conventional microscope resolution with 20x objective lens is $0.84 \mu \mathrm{m}$, which can be obtained by

$$
r=0.61 \lambda / N A
$$
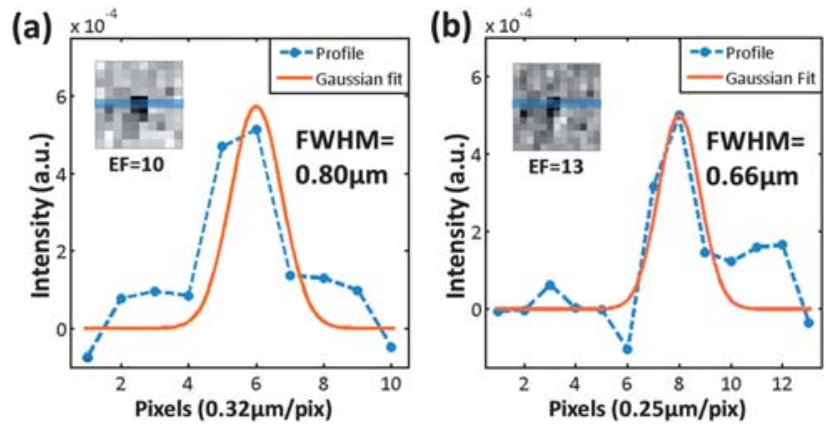

Fig. 3 Resolution of the SROFM prototype obtained with $0.5 \mu \mathrm{m}$ microspheres. (a) Intensity profile of the $0.5 \mu \mathrm{m}$ sphere from image reconstructed with an enhancement factor of 10 (inset). (b) Intensity profile of the same sphere reconstructed with an enhancement factor of 13 (inset). FWHMs of the profiles in (a) and (b) are $0.8 \mu \mathrm{m}$ and $0.66 \mu \mathrm{m}$, respectively.

where NA is the numerical aperture of the objective lens and $\lambda$ is the wavelength of light. For aperture-based OFM, $0.8 \mu \mathrm{m}$ resolution has been achieved with $1 \mu \mathrm{m}$ apertures. ${ }^{4}$ It has been shown that the resolution of aperture-based OFM is dependent on the aperture diameter and that a $450 \mathrm{~nm}$ aperture can achieve $\sim 400$ $\mathrm{nm}$ resolution. ${ }^{16}$ Resolution of SROFM can also be improved by using smaller LR pixel size and/or higher enhancement factor with longer LR sequences. A detailed study of the resolution limit with different sample-to-floor separations requires a nearfield scanning optical microscope (NSOM) measurement and will be presented elsewhere.

SROFM is not only simpler than the previous aperture-based OFM, but also more robust and able to image samples flowing with non-uniform translational motion and even rotation. Since the motion vector of a LR image sequence with SROFM is estimated from the image sequence itself, precise flow control is no longer required. Hence, whereas aperture-based OFM would
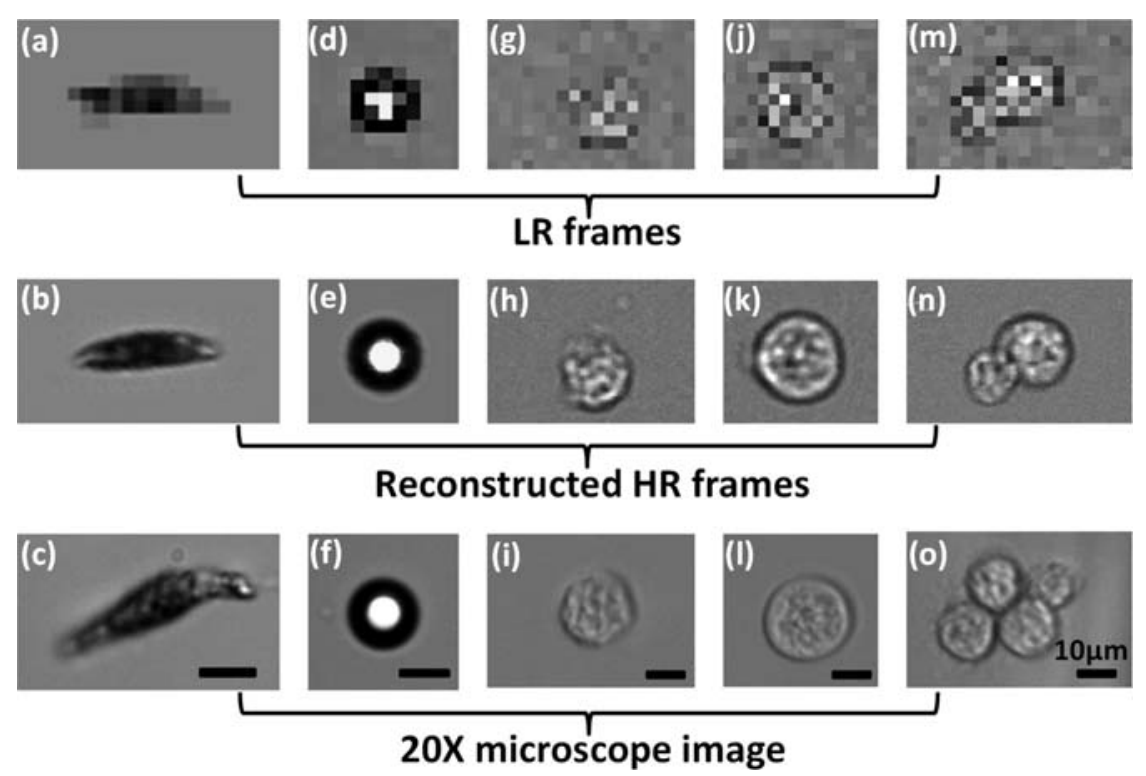

Fig. 2 Images obtained from the SROFM device. Euglena gracilis (a-c), microsphere (d-f) and Entamoeba invadens cysts (g-o). Raw low resolution (LR) images from the sensor (top row), high resolution (HR) images reconstructed from the sequence of LR images (middle row) and conventional bright field microscope images taken with 20x objective lens (bottom row). 40 to 50 LR frames were used to reconstruct each HR image. 

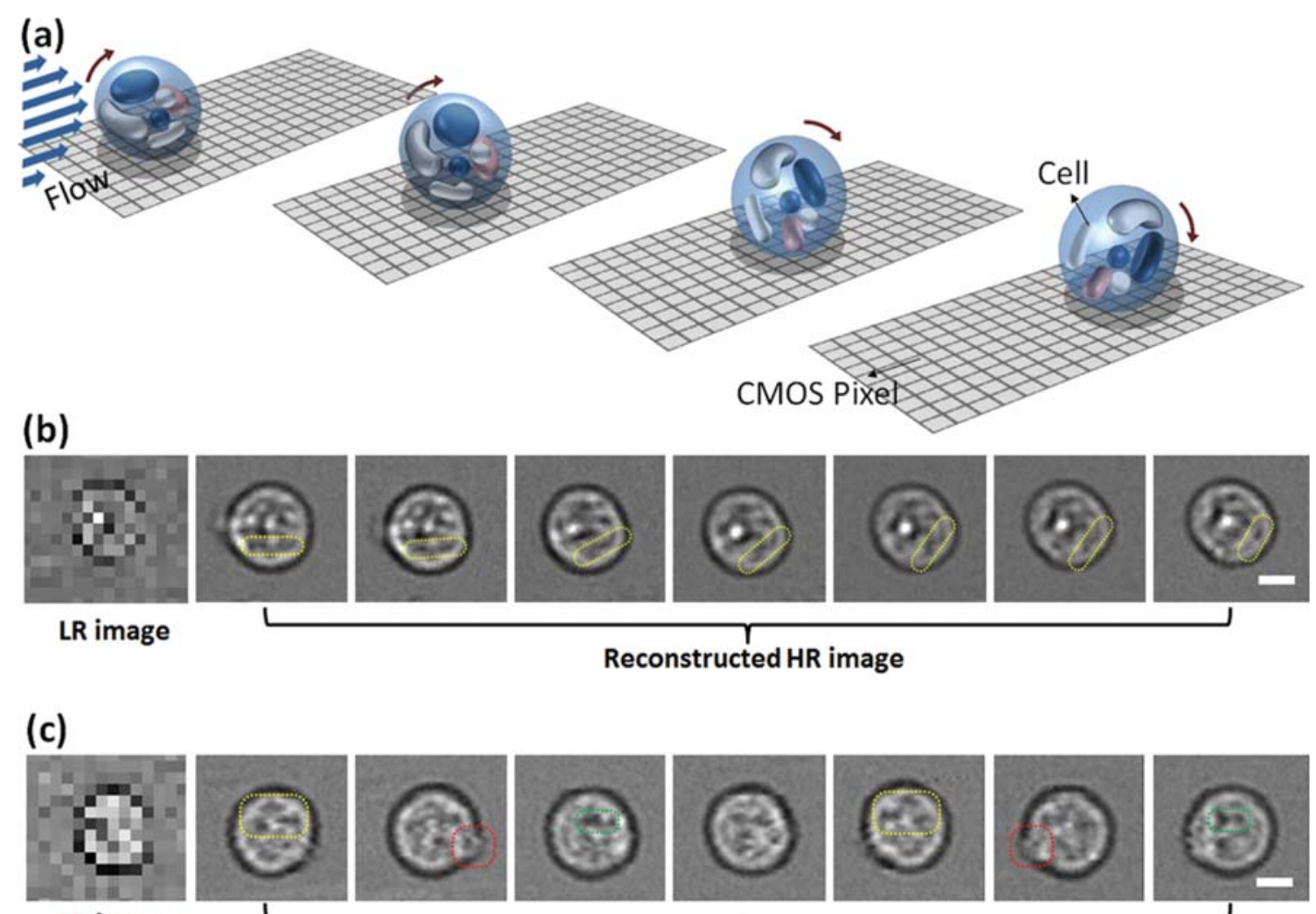

LR image
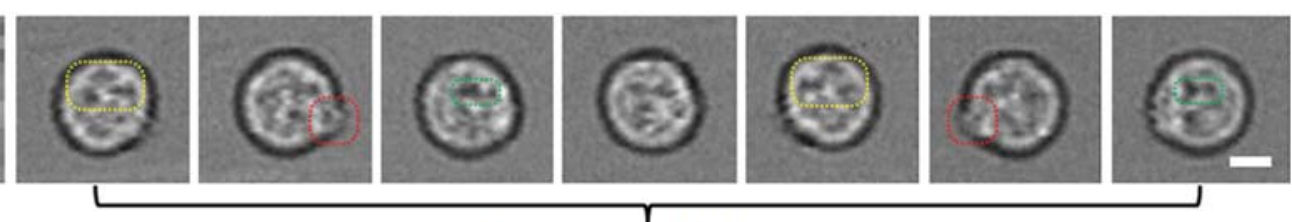

Reconstructed HR image

Fig. 4 Sequential HR images of rotating cells (scale bar $10 \mu \mathrm{m}$ ). (a) Cells flowing with rotational movement allow the SROFM to capture images of different projections, revealing the three-dimensional inner cellular structures. (b) HR sequence of an Entamoeba invadens cyst travelling with in-plane rotation. Note that the highlighted part of the sample is rotating in the sequence. The length of the dark spot in the highlighted region is also decreasing in the time lapse, indicating that the sample has out-of-plane rotation as well. (c) Sample translating with out-of-plane rotation. The highlighted regions with the same color reveal that the pairs of images are rotated 180 degrees out-of-plane of each other. Each pair of images is distinct from the others, showing the complex inner structure of the cell from a different projection plane.

utilize an additional electrokinetic driving scheme to ensure uniform flow, SROFM devices can be used with a drop-and-flow scheme, where a small volume of liquid sample is injected from the inlet and drawn into the channel by capillary action and the pressure difference between inlet and outlet. Even though the flow rate will gradually decrease as pressure between the inlet and the outlet equalizes, a non-linear motion vector can be estimated and used to reconstruct a high quality HR image. Furthermore, SROFM can image samples flowing with rotational movement, as long as the rate of rotation is slow relative to the image capture frame rate. Fig. 4(b) shows the sequence of HR images of a cell near the channel side wall, flowing with both in-plane and out-ofplane rotation.

SROFM's ability to image samples with rotational movement provides us with the unique opportunity to image samples from different perspectives. When cells rotate out-of-plane while flowing through the channel, we can observe the cells from different projections, and hence better resolve their threedimensional inner structures. The requisite rotational motion for such imaging is naturally provided by the interaction of the cell with the non-uniform flow velocity profile of pressure-driven laminar fluid flow in the microfluidic channel. Fig. 4(c) shows sequential HR images of an Entamoeba invadens cyst rolling in the channel. Note that pairs of images highlighted with the same color are mirror images of each other, indicating that the cell has rotated 180 degrees along the direction of flow. These images reveal the three-dimensional location of each of the dark and

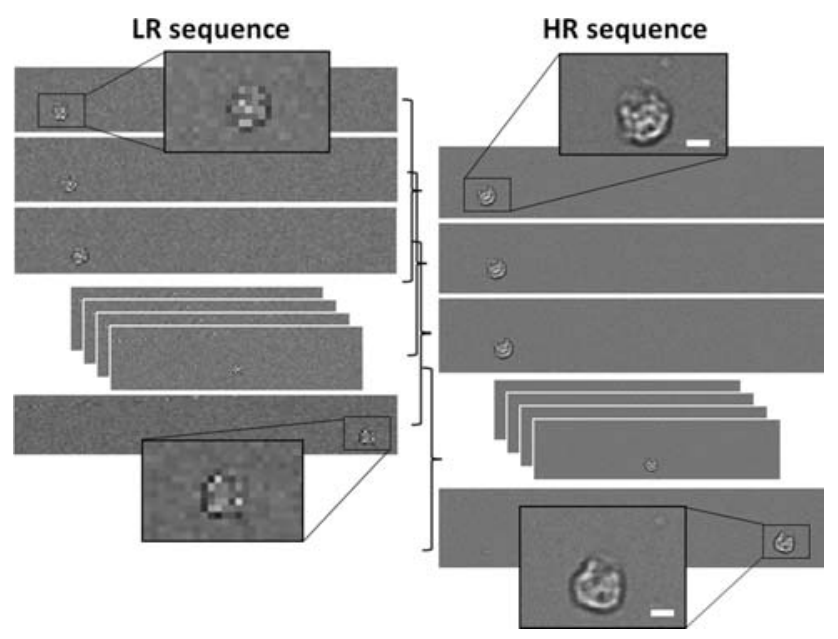

Fig. 5 Reconstruction of HR video using the multiframe pixel superresolution algorithm (scale bar $10 \mu \mathrm{m}$ ). 
bright spots in the cyst, as well as its external morphology. 350 frames of the LR image sequence were used to reconstruct the HR images, with 50 LR frames used for each HR frame. The motion blur due to the rotation within each 50 frames is not itself significant in the HR images, but if the rate of rotation of the sample is too fast relative to the translational movement, then the rotation blur would degrade the image quality. Additional modification in the design of the microfluidic channel could help control the rotation of the sample to achieve consistent rotational imaging without significant motion-blur. As such, we can perform rotational imaging with SROFM without any additional scanning stages or multiple light sources. The flexible ability of SROFM in imaging samples that are rotating or flowing with non-uniform velocities is a significant advantage over other designs.

SROFM can not only acquire HR still images of samples, but also capture HR videos of the samples as they flow through the channel and interact with the fluid. This is accomplished by using a sliding window sampling approach in combination with the pixel super-resolution algorithm (Fig. 5, see ESI for the reconstructed HR video $\dagger$ ). We demonstrate HR video of Entamoeba invadens cysts flowing within the channel. In this video, we observe multiple non-rotating cysts flowing across the channel, a single rotating cyst and a single cyst flowing with out-of-plane rotation. To reconstruct each frame of each HR video, $45 \mathrm{LR}$ frames were used with an enhancement factor $n=7$. SROFM's implementation of pixel super-resolution HR video reconstruction may find many practical applications, including the monitoring of the dynamic behavior, rotation and deformation, of a cell in a fluidic channel and creation of synthetic "video zoom", where a region of interest within a video sequence is enlarged and enhanced by some factor.

\section{Conclusions}

We proposed and demonstrated a new on-chip optofluidic microscope that utilizes a multiframe pixel super-resolution algorithm to capture and reconstruct HR images and videos of biological samples. The novel combination of a pixel superresolution and optofluidic approach towards microscopy removes the need for bulky and expensive lenses, coherent illumination sources and precision microscanning mechanisms. In addition, the SROFM system also allows us to capture images and videos of rotating samples in high resolution and, thereby, reveal three-dimensional sub-cellular structures from different perspectives.

SROFM's simplicity, compactness, and cost-effectiveness make it well-suited for drop-and-flow screening of fluidic samples such as blood, urine or water. We believe that the SROFM technique can potentially play a significant role in the eventual development and commercialization of a mass-distributed, portable microscope for point-of-care analysis and third-world diagnostics, for the detection of water-borne parasites, bloodborne parasites and diseases involving blood cell deformations.

\section{Experimental}

\section{Fabrication of SROFM device}

The device is comprised of two parts; a CMOS image sensor and a PDMS microfluidic channel. We used MT9T031C12STC (3.2 $\mu \mathrm{m}$ pixel, Aptina) for the image sensors. We removed the color filter and the microlens layer by treating the sensor under oxygen plasma for $10 \mathrm{~min}(80 \mathrm{~W})$.

The PDMS channel is fabricated by conventional soft lithography; SU-8 (SU-8 2002, 2007, 2015, Microchem) mold is fabricated on a 3 inch silicon wafer by mask photolithography. The mold is then casted by poly-dimethylsiloxane (Sylgard, Dow-corning) of $1: 10 \mathrm{mix}$ with base and curing agent, baked at $80^{\circ} \mathrm{C}$ for $1 \mathrm{~h}$. The fluidic channel is peeled, punched, and cut. The surfaces of the fluidic channel and the sensor are cleaned with ethanol and oxygen plasma, and then bonded to each other.

Polyethylene Glycol (PEG, Sigma-Aldrich) surface treatment is used to render the channel surface hydrophilic and to reduce cell adhesion. ${ }^{17} \mathrm{PEG}$ solution is injected into the channel and the device is left under $\mathrm{UV}^{4}$ lamp for $30 \mathrm{~min}$. Then, the channel is flushed with DI water and dried.

\section{Acknowledgements}

We are grateful for the constructive discussions with and generous help from Dr Jigang Wu, Mr. Lap Man Lee, Mr. Jian Ren and Mr. Shuo Pang from Caltech. We thank Dr Daniel Echinger from New York University Medical Center for sending us Entamoeba invadens cyst samples. We acknowledge funding support from the Coulter Foundation and Gates Foundation.

\section{References}

1 X. Heng, D. Erickson, L. Baugh, Z. Yaqoob, P. Sternberg, D. Psaltis and C. Yang, Lab Chip, 2006, 6, 1274.

$2 \mathrm{~W} . \mathrm{Xu}, \mathrm{M}$. Jericho, I. Meinertzhagen and H. Kreuzer, Proc. Natl. Acad. Sci. U. S. A., 2001, 98, 11301.

3 J. Garcia-Sucerquia, W. Xu, M. Jericho and H. Kreuzer, Opt. Lett., 2006, 31, 1211.

4 X. Cui, L. Lee, X. Heng, W. Zhong, P. Sternberg, D. Psaltis and C. Yang, Proc. Natl. Acad. Sci. U. S. A., 2008, 105, 10670.

5 N. Lindquist, A. Lesuffleur, H. Im and S. Oh, Lab Chip, 2009, 9, 382.

6 S. Seo, T. Su, D. Tseng, A. Erlinger and A. Ozcan, Lab Chip, 2009, 9 , 777.

7 E. Schonbrun, W. Ye and K. Crozier, Opt. Lett., 2009, 34, 2228.

8 G. Zheng, X. Cui and C. Yang, Proc. Natl. Acad. Sci. U. S. A., 2010, 107, 9043 .

9 E. Schonbrun, A. Abate and P. Steinvurzel, Lab Chip, 2010, 10, 852.

10 G. Zheng and C. Yang, Opt. Lett., 2010, 35, 2636.

11 D. Breslauer, R. Maamari, N. Switz, W. Lam, D. Fletcher and M. Pai, PLoS One, 2009, 4, e6320.

12 J. Wu, X. Cui, G. Zheng, Y. M. Wang, L. M. Lee and C. Yang, Opt. Lett., 2010, 35, 2188.

13 Y. Wang, G. Zheng and C. Yang, Opt. Express, 2009, 17, 23903.

14 W. Bishara, T. Su, A. Coskun and A. Ozcan, Opt. Express, 2010, 18, 11181.

15 M. Elad and Y. Hel-Or, IEEE Trans. Image Process., 2001, 10, 1187.

16 X. Heng, X. Cui, D. W. Knapp, J. Wu, Z. Yaqoob, E. J. McDowell, D. Psaltis and C. Yang, Opt. Express, 2006, 14, 10410.

17 S. Hu, X. Ren, M. Bachman, C. Sims, G. Li and N. Allbritton, Anal. Chem., 2002, 74, 4117. 